\title{
Briófitas da Ilhabela, Estado de São Paulo, Brasil
}

\author{
Olga Yano ${ }^{1}$ e Denilson Fernandes Peralta ${ }^{1,2}$
}

Recebido: 22.03.2007; aceito: 27.03.2008

\begin{abstract}
Bryophytes from Ilhabela, São Paulo State, Brazil). Sixty eigthy bryophyte taxa distributed in 49 genera and 35 families were identify to Ilhabela. Among them two species are hornworts, 35 mosses and 31 liverworts. The most richeness families in moss are Pilotrichaceae, Pottiaceae and Sematophyllaceae, with 3 species each, Fissidentaceae, Hypnaceae, Orthotrichaceae and Polytrichaceae with 2 species each, in liverworts Lejeuneaceae 13 species, Jubulaceae 5, Plagiochilaceae 4 and Radulaceae with 3 each. Callicostella ciliata (Schimp. ex Besch.) A. Jaeger and Meiothecium boryanum (Müll. Hal.) Mitt. are new records to São Paulo State.
\end{abstract}

Key words: Ilhabela, liverworts, mosses, São Paulo State

RESUMO - (Briófitas da Ilhabela, Estado de São Paulo, Brasil). Foram identificadas 68 espécies de briófitas para a Ilhabela, distribuídas em 49 gêneros e 35 famílias, sendo dois antóceros, 35 musgos e 31 hepáticas. As famílias mais ricas de musgos foram Pilotrichaceae, Pottiaceae e Sematophyllaceae, todas com três espécies cada uma, seguida de Fissidentaceae, Hypnaceae, Orthotrichaceae e Polytrichaceae com duas espécies cada uma. Para as hepáticas as famílias mais ricas foram Lejeuneaceae com 13 espécies, Jubulaceae com cinco, Plagiochilaceae com quatro e Radulaceae com três espécies. Todas as demais famílias apresentaram uma espécie cada. Callicostella ciliata (Schimp. ex Besch.) A. Jaeger e Meiothecium boryanum (Müll. Hal.) Mitt. são novas ocorrências para o estado de São Paulo. Palavras-chave: Ilhabela, hepáticas, musgos, São Paulo

\section{Introdução}

A Ilhabela está localizada no município de

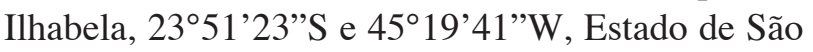
Paulo, a $210 \mathrm{~km}$ da capital. Possui uma área de 340 $\mathrm{km}^{2}$ e dista $2,5 \mathrm{~km}$ do continente. Cerca de $85 \%$ do município de Ilhabela pertence ao Parque Estadual da Ilhabela, juntamente com a Ilha de São Sebastião, as ilhas de Búzios, da Vitória e outras que integram a rede de Unidades de Conservação administrada pelo Instituto Florestal da Secretaria do Meio Ambiente de São Paulo.

A ilha é dividida fisicamente a partir do píer de chegada em duas porções: Norte e Sul. é predominantemente coberta pela vegetação formada por Mata Atlântica com áreas antropizadas e afloramentos rochosos. O terreno é acidentado e os picos de montanhas, cobertos de floresta, atingem ca. de 1.300 metros de altitude.

Existem poucos estudos em Ilhas, principalmente, no estado de São Paulo, fato já enfatizado por Ângelo (1989). Em relação às briófitas, foram encontrados em literatura registros de estudos em sete ilhas do estado de São Paulo, o que corresponde a 6,6\% das 106 ilhas existentes (Ângelo 1989).

Para as ilhas do estado de São Paulo os estudos realizados são os de: Yano (1990) registrou oito espécies de briófitas no Parque Estadual da Ilha do Cardoso. Visnadi \& Vital (2001) realizaram estudo das briófitas das Ilhas de Alcatrazes, Bom Abrigo, da Casca e do Castilho, encontrando 61 espécies. Recentemente Yano et al. (2003) encontraram 12 espécies de musgos ao inventariar as briófitas da Ilha Urubuqueçaba, no município de Santos, Peralta (2005) estudou os musgos da Ilha Anchieta registrando 129 táxons e finalmente Joyce et al. (2006) que listaram 36 espécies de briófitas para a Ilha das Palmas, no município de Guarujá. No total estes trabalhos listam 331 espécies de briófitas para as ilhas do estado de São Paulo. As espécies encontradas em ilhas parecem não diferir das espécies continentais, entretanto ainda são necessários estudos taxonômicos e ecológicos para se conhecer a dinâmica das populações de briófitas e ambientes insulares.

1. Instituto de Botânica, Caixa Postal 3005, 01061-970 São Paulo, Brasil

2. Autor para correspondência: denilsonfp@yahoo.com.br 
O objetivo deste trabalho foi ampliar o levantamento das espécies de briófitas das ilhas do Estado de São Paulo.

\section{Material e métodos}

Para a realização deste trabalho foram utilizadas as amostras depositadas no Herbário Científico do Estado "Maria Eneyda P. Kauffmann Fidalgo", do Instituto de Botânica (SP), coletadas em três excursões realizadas nos períodos de outubro/1977, dezembro/1982 e junho/1983 e outras amostras. No total foram analisadas 98 amostras de briófitas.

As identificações basearam-se nos trabalhos de Florschütz (1964), Fulford (1968, 1976), Bischler (1969), Schuster (1980), Reese (1993), Gradstein (1994), Sharp et al. (1994), Churchill \& Linares C. (1995), Buck (1998), Oliveira-e-Silva \& Yano (2000) e Gradstein \& Costa (2003).

Os sistemas de classificação adotados foram os de Stotler \& Crandall-Stotler (2005) para Anthocerotophyta, Buck \& Goffinet (2000) para Bryophyta, com modificação na família Leucobryaceae (La Farge et al. 2000), e CrandallStotler \& Stotler (2000) para Marchantiophyta. Os táxons são apresentados em ordem alfabética de família, gênero e espécies, o “*”, indica as novas ocorrências para o estado.

\section{Resultados e Discussão}

Foram identificadas 68 espécies de briófitas e uma variedade, pertencentes a 49 gêneros e 35 famílias, sendo dois antóceros, 35 musgos e 31 hepáticas. Este número representa $20,5 \%$ do total de espécies conhecidas para ilhas no litoral paulista
(331 espécies) e 2,3\% das 2.961 espécies conhecidas para o Brasil (Yano \& Peralta 2007).

As famílias de musgos mais ricas foram Pilotrichaceae, Pottiaceae e Sematophyllaceae, todas com três espécies cada, seguidas de Fissidentaceae, Hypnaceae, Orthotrichaceae e Polytrichaceae com duas espécies cada. Para as hepáticas as famílias mais ricas foram Lejeuneaceae com 13 espécies, Jubulaceae com cinco, Plagiochilaceae com quatro e Radulaceae com três espécies. Todas as demais famílias apresentaram uma espécie cada.

A maioria das espécies encontradas neste levantamento apresenta ampla distribuição brasileira e neotropical. Apenas as novas ocorrências para o Estado de São Paulo, Callicostella cilliata (Schimp. ex Besch.) A. Jaeger e Meiothecium boryanum (Müll. Hal.) Mitt., apresentam distribuição mais restrita no Brasil.

A localização geográfica da Ilhabela na região Sudeste do Brasil condicionada pela grande umidade e relevo acidentado proporciona grande riqueza de espécies de briófitas. Dessa maneira a riqueza de espécies encontrado é alta e diversificada. Entre as ilhas do litoral paulista apenas a Ilha Anchieta possui maior número de táxons que a Ilhabela (tabela 1), devido a ter sido realizado um trabalho de coletas sistemático na Ilha Anchieta que resultou em uma dissertação de mestrado.

As espécies encontradas, especialmente as espécies pequenas da família Lejeuneaceae, e o gênero Trichocolea mostram que a área é preservada. Algumas espécies comuns, como Bryum argenteum Hedw. ou qualquer espécie de Campylopus ou da família Lepidoziaceae, não foram encontradas neste levantamento, porém provavelmente ocorrem na área, o que mostra a necessidade de outros estudos taxonômicos e ecológicos das briófitas na área.

Tabela 1. Comparação entre a riqueza de briófitas e a área das ilhas estudadas no litoral paulista.

\begin{tabular}{llrr}
\hline Localidade & Referência & $\mathrm{n}^{\circ}$ sp. & Área $\left(\mathrm{km}^{2}\right)$ \\
\hline Ilha do Cardoso & Yano (1990) & 8 & 300,00 \\
Ilha Urubuqueçaba & Yano et al. (2003) & 23 & 2,00 \\
Ilha do Castilho, Ilha da Casca, Ilha de & Visnadi \& Vital (2001) & 61 & 3,56 \\
Alcatrazes, Ilha do Bom Abrigo & & 244 & 8,28 \\
Ilha Anchieta & Peralta \& Yano (dados não publicados) & 68 & 340,00 \\
Ilhabela & este estudo & &
\end{tabular}




\section{Anthocerotophyta}

\section{ANTHOCEROTACEAE}

Anthoceros punctatus L., Sp. Pl. 2: 1139. 1753.

Material examinado: sobre o barranco úmido, perto do riacho grande, 27-VII-1983, O. Yano et al. 7968 (SP189417).

Distribuição no Brasil: AM, BA, CE, RJ, RS, SC e SP.

\section{NOTOTHYLADACEAE}

Phaeoceros laevis (L.) Prosk., Bull. Torrey Bot. Club 78(4): 346. 1951 三 Anthoceros laevis L., Sp. Pl. 2: 1139.1753.

Material examinado: estrada sul, na margem do rio perto da Praia Grande, 29-IX-1977, O. Yano 860 (SP132612); idem, caminho para cachoeira da Toca, sobre pedra, margem do riacho, 31-XII-1982, $O$. Yano 5118 p.p. (SP181042); idem, estrada sul, perto do riacho grande, 27-VII-1983, O. Yano et al. 7967 (SP189416); idem, no barranco úmido formando um grande tapete amarelo, 27-VII-1983, O. Yano et al. 7971 (SP189420).

Distribuição no Brasil: AM, BA, DF, ES, GO, MG, MS, PE, RJ, RS, SC e SP. Associada a Sematophyllum galipense (Müll. Hal.) Mitt.

\section{Bryophyta}

\section{BARTRAMIACEAE}

Philonotis hastata (Duby) Wijk \& Marg., Taxon 8: 74. 1959 = Hypnum hastatum Duby in Moritzi, Syst. Verz. Zool. Pfl. Java.: 132. 1846.

Material examinado: estrada norte, perto da Bica da água, às vezes quase submerso, 29-IX-1977, O. Yano 870 (SP136112).

Distribuição no Brasil: AM, CE, GO, MS, MT, PA, PR, RJ, RO, RS e SP.

\section{BRACHYTHECIACEAE}

Meteoridium remotifolium (Müll. Hal.) Manuel, Lindbergia 4(1-2): 49-51.1977 $\equiv$ Leskea remotifolia Müll. Hal., Linnaea 19: 216. 1846.

Material examinado: cachoeira do Toca, sobre húmus na pedra úmida, 31-XII-1982, O. Yano 5130
(SP172561); idem, sobre galhos e troncos perto da cachoeira da Toca, 31-XII-1982, O. Yano 5144 (SP172566).

Distribuição no Brasil: BA, ES, GO, MG, MT, PB, P,E, PR, RJ, RR, RS, SC e SP.

\section{BRYACEAE}

Bryum grandifolium (Tayl.) Müll. Hal., Syn. Musc. Frond. 1: 250. 1848 = Mnium grandifolium Tayl., London J. Bot. 6: 336. 1847.

Material examinado: sobre húmus em pedras grandes na vegetação de Eucalyptus, 29-IX-1977, O. Yano 867 p.p. (SP136642); idem, estrada sul, ca. $20 \mathrm{~km} \mathrm{da}$ cidade, nas frestas da pedra caindo água, 31-XII-1982, O. Yano 5167 (SP172579).

Distribuição no Brasil: BA, MT, RJ, RO, SC e SP. Associada a Octoblepharum albidum Hedw.

\section{CALYMPERACEAE}

Calymperes palisotii Schwäegr., Spec. Musc. Suppl. 1(2): 334. 1816.

Material examinado: sobre tronco de jaqueira, 29-IX-1977, O. Yano 855 p.p. (SP136101); idem, praça Coronel Julião Moura Negrão, sobre tronco de Delonnis regia, 31-XII-1982, O. Yano 5107 p.p. (SP172553).

Distribuição no Brasil: AL, AM, AP, BA, CE, ES, FN, GO, MT, PA, PB, PE, PI, RJ, RN, RO, RR, SE e SP. Associada a Frullania ericoides (Nees) Mont., $F$. neesii Lindenb. e Schiffneriolejeunea polycarpa (Nees) Gradst.

\section{DICRANACEAE}

Holomitrium olfersianum Hornsch., Fl. Bras. 1(2): 8. 1840.

Material examinado: estrada sul, ca. $20 \mathrm{~km}$ da cidade, sobre pedras grandes junto ao rio, 31-XII-1982, $O$. Yano 5163 (SP172577).

Distribuição no Brasil: BA, ES, MG, MT, PR, RJ, $\mathrm{RS}, \mathrm{SC}$ e SP.

\section{ERPODIACEAE}

Erpodium glaziovii Hampe, Vidensk. Meddr. Dansk. Naturh. Foren. Kjøbenhavn. ser. 3, 4: 54. 1872. 
Material examinado: praça Coronel Julião Moura Negrão, sobre tronco de Croton sp., 31-XII-1982, O. Yano 5109 (SP172554).

Distribuição no Brasil: AM, BA, ES, MG, MS, PR, RJ, SC e SP.

\section{FISSIDENTACEAE}

Fissidens asplenioides Hedw., Spec. Musc. Frond.: 156. 1801.

Material examinado: estrada sul, na margem do rio, perto de uma árvore com respingos de água, 29-IX-1977, O. Yano 857 (SP136102); idem, cachoeira da Toca, sobre pedra úmida, junto da cachoira e corredeiras de água, 31-XII-1982, O. Yano 5150 (SP172570).

Distribuição no Brasil: BA, ES, MG, PR, RJ, RR, RS, SC e SP.

Fissidens zollingeri Mont., Ann. Sci. Nat. Bot. ser 3, 4: 114. 1845.

Material examinado: estrada norte, no solo arenoso, vegetação de Eucalyptus sp. perto da Praia dos Sinus, 29-IX-1977, O. Yano 863 (SP136106).

Distribuição no Brasil: AC, AM, AP, BA, CE, ES, FN, GO, MA, MG, MS, MT, PA, PB, PE, PI, PR, RJ, RO, RR, SC, SP e TO.

\section{HELICOPHYLLACEAE}

Helicophyllum torquatum (Hook.) Brid., Bryol. Univ. 2: 771.1827 = Anictangium torquatum Hook., Musci Exot. 1: 41. 1818.

Material examinado: sobre pedras grandes, a $50 \mathrm{~cm} \mathrm{da}$ água do mar, 29-IX-1977, O. Yano 854 (SP136100); idem, estrada sul, sobre tronco de jaqueira, perto da Praia Grande, 29-IX-1977, O. Yano 861 (SP136104); idem, estrada norte, ca. $18 \mathrm{~km}$ da cidade, sobre bloco de pedra, na mata, 31-XII-1982, O. Yano 5111 (SP172556); idem, estrada norte, ca. $5 \mathrm{~km}$ do centro da cidade, sobre tronco vivo de Eucalyptus sp., 27-VII-1983, O. Yano et al. 7961 (SP189410); idem, estrada sul, perto do riacho grande, sobre pedras e troncos, mata ciliar, 27-VII-1983, O. Yano et al. 7966 (SP189415).

Distribuição no Brasil: AL, AM, BA, CE, DF, ES, GO, MG, MS, MT, PA, PB, PE, PI, PR, RJ, SC, SP e TO.

\section{HYPNACEAE}

Chryso-hypnum diminutivum (Hampe) W.R. Buck, Brittonia 36: 182. 1984 三 Hypnum diminutivum Hampe, Linnaea 20: 86. 1847.

Material examinado: estrada sul, perto do riacho Grande, sobre pedras no leito do riacho, 27-VII-1983, O. Yano et al. 7970 (SP189419).

Distribuição no Brasil: AC, AM, AP, DF, ES, GO, MG, MS, MT, PA, PE, PR, RJ, RR, RS, SC e SP.

Vesicularia vesicularis (Schwägr.) Broth. in Engler \& Prantl, Natürl. Pflanzenfam. 1 (3): 1094. 1908 三 Hypnum vesiculare Schwägr., Spec. Musc. Frond. Suppl. 2, 2: 167. 1827.

Material examinado: cachoeira do Toca, sobre tronco úmido, 31-XII-1982, O. Yano 5149 (SP172569).

Distribuição no Brasil: AC, AM, ES, MG, MS, MT, PA, PE, PI, RJ, RO, RR, RS, SC e SP.

Vesicularia vesicularis (Schwägr.) Broth. var. vesicularis, Naturl. Pflanzenfam. 1 (3): 1094. $1908 \equiv$ Hypnum vesiculare Schwägr., Spec. Musc. Frond., Suppl. 2, 2: 167. 1827.

Material examinado: sobre pedras nos nas margens de um riacho, 5-1-1971, D.M. Vital 1735 p.p. (SP87561); idem, estrada sul, ca. $20 \mathrm{~km}$ da cidade, sobre pedras semicobertas pela água do rio, 31-XII-1982, O. Yano et al. 5168 p.p. (SP181069).

Distribuição no Brasil: AM, MS, PR, SC e SP. Associada a Dumortiera hirsuta (Sw.) Nees e Marchantia chenopoda $\mathrm{L}$.

\section{HYPOPTERYGIACEAE}

Hypopterygium tamarisci (Sw.) Brid. ex Müll. Hal., Syn. Musc. Frond. 2: 8. $1850 \equiv$ Hypnum tamarisci Sw., Fl. Ind. Occ. 3: 1825. 1806.

Material examinado: cachoeira da Toca, sobre pedra úmida, 31-XII-1982, O. Yano 5131 (SP172562).

Distribuição no Brasil: BA, ES, MG, PR, RJ, RS, SC e SP.

\section{LEUCOBRYACEAE}

Leucobryum clavatum Hampe, Vidensk. MeddeI. Dansk Naturhist. Foren. Kjöbenhavn, sér. 3, 9-10: 252. 1877. 
Material examinado: sobre tronco morto na margem de um riacho, 17-X-1970, D.M. Vital 1736 (SP90111).

Distribuição no Brasil: DF, MG, MT, PR, RJ, RS, SC e SP.

\section{MYRINIACEAE}

Helicodontium capillare (Hedw.) A. Jaeger, Ber. Thätigk. St. Gallischen Naturwiss. Ges. 1876-77: 225.1878 =Leskea capillaris Hedw., Spec. Musc. Frond.: 221. 1801.

Material examinado: estrada sul, perto do riacho Grande, na base do tronco vivo, na mata ciliar, 27-VII-1983, O. Yano et al. 7969 p.p. (SP189418).

Distribuição no Brasil: BA, ES, GO, MG, PR, RJ, RS, SC e SP. Associada a Racopilum tomentosum (Hedw.) Brid. e Stereophyllum radiculosum (Hook.) Mitt.

\section{NECKERACEAE}

Neckeropsis disticha (Hedw.) Kindb., Canad. Rec. Sci. 6: 21. 1894 = Neckera disticha Hedw., Spec. Musc. Frond.: 201. 1801.

Material examinado: margem do riacho Grande, na base do tronco vivo, mata ciliar, 27-VII-1983, O. Yano et al. 7962 (SP189411).

Distribuição no Brasil: AC, AM, BA, ES, GO, MG, MT, P A, PE, PR, RJ, RR, RS, SC e SP.

Porotrichum longirostre (Hook.) Mitt., J. Linn. Soc. Bol. 12: 462.1869 = Neckera longirostre Hook., Musci Exot. 1: 1. 1818.

Material examinado: cachoeira do Toca, sobre pedra junto da corredeira de água, 31-XII-1982, O. Yano 5141 (SP172564).

Distribuição no Brasil: MG, MS, MT, RJ, RS, SC e SP.

\section{OCTOBLEPHARACEAE}

Octoblepharum albidum Hedw., Spec. Musc. Frond.: 50. 1801.

Material examinado: estrada norte, na base do tronco de Eucalyptus perto da praia dos Sinos, 29-IX-1977, O. Yano 865 (SP136108); idem, sobre húmus em pedras grandes na vegetação de Eucalyptus, 29-IX-1977, O. Yano 867 p.p., 868 (SP136642, SP136110); idem, estrada do sul, ca. $10 \mathrm{~km}$ da cidade, sobre blocos de pedra, 31-XII-1982, O. Yano 5151 (SP172571).
Distribuição no Brasil: AC, AL, AM, AP, BA, CE, DF, ES, FN, GO, MA, MG, MS, MT, PA, PB, PE, PI, PR, RJ, RN, RO, RR, RS, SC, SE, SP e TO. Associada a Bryum grandifolium (Tayl.) Müll. Hal.

\section{ORTHOTRICHACEAE}

Groutiella apiculata (Hook.) Crum \& Steere, Bryologist 53(2): 146. 1950 三 Orthotrichum apiculatum Hook., Musci Exot. 1: 45. 1818.

Material examinado: 31-XII-1982, O. Yano 5126 p.p., 5148 (SP181049, SP172568); idem, estrada sul, ca. $20 \mathrm{~km}$ da cidade, sobre pedras grandes junto do rio, 31-XII-1982, O. Yano 5157 (SP172574).

Distribuição no Brasil: BA, ES, MS, MT, PA, PE, PR, RJ, SC e SP. Associada a Cheilolejeunea rigidula (Mont.) R.M. Schust.

Macrocoma orthotrichoides (Raddi) Wijk \& Marg., Taxon 11: 221. $1962 \equiv$ Lasia orthotrichoides Raddi, Critt Brasil.: 6. 1822.

Material examinado: estrada norte, ca. $10 \mathrm{~km}$ da cidade sobre tronco vivo de Leguminosae, 31-XII-1982, $O$. Yano 5113 (SP172557).

Distribuição no Brasil: BA, ES, MG, PR, RJ, RS, SC e SP.

\section{PHYLLOGONIACEAE}

Phyllogonium viride Brid., Bryol. Univ. 2: 673. 1827.

Material examinado: estrada sul, ca. $20 \mathrm{~km}$ da cidade, sobre pedras grandes junto do rio, 31-XII-1982, $O$. Yano 5158 (SP172575).

Distribuição no Brasil: AL, BA, CE, ES, MG, PE, PR, RJ, RS, SC e SP.

\section{PILOTRICHACEAE}

* Callicostella ciliata (Schimp. ex Besch.) A. Jaeger, Ber. Thätigk. St. Gallischen Naturwiss. Ges. 1875-76: 355. 1877.

Material examinado: estrada sul, ca. $20 \mathrm{~km}$ da cidade, sobre pedra submersa na água do rio, 31-XII-1982, O. Yano 5164 (SP172578).

Distribuição no Brasil: MT e primeira ocorrência para São Paulo.

Callicostella pallida (Hornsch.) Ångstr., Öfvers. Förh. Kongl. Svenska VetenskAkad. 33(4): 27. 1876 三 
Hookeria pallida Hornsch. in Martius, Flora Brasil. 1 (2): 64.1840.

Material examinado: cachoeira do Toca, sobre pedra úmida, 31-XII-1982, O. Yano 5143 (SP172565).

Distribuição no Brasil: AC, AM, AP, BA, CE, DF, ES, GO, MG, MS, MT, PA, PE, PR, RJ, RO, RR, RS, SE, SP e TO. Associada a Thuidium delicatulum (Hedw.) Bruch \& Schimp.

Thamniopsis incurva (Hornsch.) W.R. Buck, Brittonia 39: 218. 1987 = Chaetophora incurva Hornsch. in Nees, Horae Phys. Berol.: 65. 1820.

Material examinado: cachoeira do Toca, sobre pedra perto da margem, 31-XII-1982, O. Yano 5124 (SP172560).

Distribuição no Brasil: CE, ES, MG, PA, PR, RJ, RS, $\mathrm{SC}$ e SP.

\section{POLYTRICHACEAE}

Polytrichum commune Hedw., Spec. Musc. Frond.: 88. 1801.

Material examinado: estrada sul, ca. $15 \mathrm{~km}$ da cidade, no barranco úmido, junto da estrada, 31-XII-1982, $O$. Yano 5156 (SP172573).

Distribuição no Brasil: AM, BA, DF, GO, MG, PR, PR, RJ, RR, RS, SC e SP.

Polytrichum juniperinum Willd. ex Hedw., Spec. Musc. Frond.: 89. 1801.

Material examinado: estrada sul, ca. $15 \mathrm{~km}$ da cidade, no barranco ao sol, junto da estrada, 31-XII-1982, $O$. Yano 5155 (SP172572).

Distribuição no Brasil: BA, DF, ES, MG, PR, RJ, RR, RS, SC e TO.

\section{POTTIACEAE}

Hyophila involuta (Hook.) A. Jaeger, Ber. Thätigk. St. Gallischen Naturwiss. Ges. 1871-72: $354.1873 \equiv$ Gymnostomum involutum Hook., Musci Exot. 2: 154. 1819.

Material examinado: estrada norte, na base do tronco de Eucalyptus perto da Praia dos Sinos, 29-IX-1977, O. Yano 864 (SP136107); idem, $O$. Yano 866 (SP136109); idem, perto da Bica de água, às vezes quase submersa, 29-IX-1977, O. Yano 869 (SP136111).
Distribuição no Brasil: AM, BA, DF, ES, GO, MS, MT, PA, PB, PE, PI, PR, RJ, RO, RS e SP.

Hyophiladelphus agrarius (Hedw.) R.H. Zander, Bryologist 98(3): 372. 1995 三 Barbula agraria Hedw., Spec. Musc. Frond.: 116. 1801.

Material examinado: estrada sul, sobre blocos de pedra, perto da Praia Grande, 29-IX-1977, O. Yano 862 (SP136105).

Distribuição no Brasil: AC, AM, BA, CE, DF, FN, MG, MT, PA, PB, PE, RJ, RO, SE, SP e TO.

Tortella humilis (Hedw.) Jenn., Mann. Moss. W. Pennsylv. 96: 13. 1913 三 Barbula humilis Hedw., Spec. Musc. Frond.: 116. 1801.

Material examinado: estrada Norte, $\pm 5 \mathrm{~km}$ do centro da cidade, sobre tronco de Eucalyptus sp., 27-VII-1983, o. Yano et al. 7960 p.p. (SP189409).

Distribuição no Brasil: BA, DF, ES, GO, MG, MS, PR, RJ, RS, SC e SP. Associada a Stereophyllum radiculosum (Hook.) Mitt.

\section{RACOPILACEAE}

Racopilum tomentosum (Hedw.) Brid., Bryol. Univ. 2: 719.1827 इ Hypnum tomentosum Sw. ex Hedw., Spec. Musc. Frond.: 240.1801.

Material examinado: estrada sul perto do riacho Grande, na base do tronco vivo, mata ciliar, 27-VII1983, O. Yano et al. 7969 p.p. (SP189418).

Distribuição no Brasil: AC, AM, BA, CE, DF, ES, GO, MG, MS, MT, P A, PE, PR, RJ, RO, RS, SC e SP. Associada a Helicodontium capillare (Hedw.) Jenn. e Stereophyllum radiculosum (Hook.) Mitt.

\section{RHIZOGONIACEAE}

Pyrrhobryum spiniforme (Hedw.) Mit1., J. Linn. Soc. Bol. 10: 174. 1868 इ Hypnum spiniforme Hedw., Spec. Musc. Frond.: 236. 1801.

Material examinado: sobre tronco na margem de um riacho, 5-I-1970, D.M. Vital 1737 (SP90112); idem, cachoeira da Toca, sobre paredão úmido, 31-XII-1982, O. Yano 5136 (SP172563).

Distribuição no Brasil: AM, BA, ES, MG, MT, PE, PR, RJ, RO, RR, RS, SC e SP. 


\section{SEMATOPHYLLACEAE}

*Meiothecium boryanum (Müll. Hal.) Mitt., J. Linn. Soc., Bot. 12: 469.1869 = Neckera boryana Müll. Hal., Syn. Musc. Frond. 2: 75. 1850.

Material examinado: estrada sul, ca. $20 \mathrm{~km}$ da cidade, sobre pedras grandes junto do rio, 31-XII-1982, $O$. Yano 5159 (SP172576).

Distribuição no Brasil: AM, DF, PA e primeira ocorrência para São Paulo.

Sematophyllum galipense (Müll. Hal.) Mitt., J. Linn. Soc. Bot. 12: 480. $1869 \equiv$ Hypnum galipense Müll. Hal., Bol. Zeitg. 6: 780. 1848.

Material examinado: estrada sul, perto do riacho Grande na base das pedras no leito do riacho, 27-VII-1983, O. Yano 7967 p.p. (SP189416).

Distribuição no Brasil: BA, CE, DF, ES, GO, MG, MT, PA, PE, PR, RJ, RO, RR, RS, SC, SP e TO. Associada a Phaeoceros laevis (L.) Prosk.

Sematophyllum subpinnatum (Brid.) Britt., Bryologist 21(2): 28. 1918 (1919) $\equiv$ Leskea subpinnata Brid., Muscol. Recent. Suppl. 2: 54. 1812.

Material examinado: caminho para cachoeira da Toca, sobre pedra, margem do riacho, 31-XII-1982, $O$. Yano 5115 (SP172559); idem, sobre tronco de árvore, 31-XII-1982, O. Yano 5147 (SP172567); idem, estrada sul, ca. $20 \mathrm{~km}$ da cidade, sobre pedra submersa na água do rio, 31-XII-1982, O. Yano 5166 p.p. (SP181068); idem, perto do riacho Grande, no barranco úmido junto da estrada, 27-VII-1983, O. Yano et al. 7963 p.p. (SP189412).

Distribuição no Brasil: AC, AM, AP, BA, CE, DF, ES, GO, MG, MS, MT, PA, PB, PE, PR, RJ, RO, RR, RS, $\mathrm{SC}, \mathrm{SP}$ e TO. Associada a Lejeunea flava (Sw.) Nees e Riccardia regnellii (Ångstr.) Hell.

\section{STEREOPHYLLACEAE}

Stereophyllum radiculosum (Hook.) Mitt., J. Linn. Soc. Bot. 12: 542. 1869 = Hookeria radiculosa Hook., Musci Exot. 1: 51. 1818.

Material examinado: estrada norte, ca. $5 \mathrm{~km}$ do centro da cidade, sobre tronco vivo de Eucalyptus sp., 27-VII-1983, O. Yano et al. 7960 p.p. (SP189409); idem, no barranco úmido, mata ciliar, 27-VII-1983, O. Yano et al. 7965 (SP189414); idem, estrada sul perto do riacho Grande, na base do tronco vivo, 27-VII-1983, O. Yano et al. 7969 p.p. (SP189418).
Distribuição no Brasil: BA, ES, GO, MG, MS, MT, P A, PR, RS, SC e SP. Associada a Helicodontium capillare (Hedw.) Jenn., Racopilum tomentosum (Hedw.) Brid. e Tortella humilis (Hedw.) Jenn.

\section{THUIDIACEAE}

Thuidium delicatulum (Hedw.) B.S.G., Bryol. Eur. 5(49/51): 164. 1852 三Hypnum delicatulum Hedw., Spec. Musc. Frond.: 260. 1801.

Material examinado: estrada sul, na margem do rio, na base da árvore, 29-IX-1977, O. Yano 858 (SP136103); idem, sobre pedra, margem do riacho, caminho para cachoeira da Toca, 31-XII-1982, O. Yano 5114 (SP172558); idem, 31-XII-1982, O. Yano 5143 p.p. (SP172565).

Distribuição no Brasil: AM, MG, MT, PA, RJ, RS e SP. Associada a Callicostella pallida (Hornsch.) Ångstr.

\section{Marchantiophyta}

\section{ANEURACEAE}

Riccardia regnellii (Ångstr.) Hell, Bolm Fac. Filos. Ciênc. S. Paulo, Bot. 25: 110. $1969 \equiv$ Pseudoneura regnellii Ångstr., Öfvers. Förh. Kongl. Svenska Vetensk.-Akad. 7: 89.1876.

Material examinado: estrada do Sul, ca. $20 \mathrm{~km}$ da cidade, sobre pedra submersa no rio, 31-XII-1982, O. Yano 5166 p.p. (SP181068).

Distribuição no Brasil: ES, MG, MT, PE, RJ, RS, SC e SP. Associada a Sematophyllum subpinnatum (Brid.) Britt.

\section{GEOCALYCACEAE}

Lophocolea martiana Nees in Gottsche et al., Syn. Hepat.: 152. 1845.

Material examinado: caminho para cachoeira da Toca, sobre pedra, na margem do riacho, 31-XII-1982, $O$. Yano 5117 p.p. (SP181041).

Distribuição no Brasil: AM, AP, BA, CE, ES, GO, MO, MT, PA, PE, PR, RJ, RS, SC, SE e SP. Associada a Symphyogyna aspera Steph. ex Evans.

\section{JUBULACEAE}

Frullania brasiliensis Raddi, Soc. Ital. Atti. Sci. Mod. 19: 36. 1822. 
Material examinado: sobre tronco de árvore na margem do riacho, 17-X-1970, D.M. Vital 1734 (SP87560).

Distribuição no Brasil: BA, CE, DF, ES, GO, MG, PE, RJ, RS, SC e SP.

Frullania ericoides (Nees) Mont., Ann. Sci. Nat. Bot. ser. 2, 12: 51. 1839 三 Jungermannia ericoides Nees in Martius, Flora Brasil. enum. plant. 1(1): 346. 1833 .

Material examinado: sobre pedra na margem de um regato, 23-1-1965, D.M. Vital 319 (SP86561); idem, praça Coronel Julião Moura Negrão, sobre tronco vivo de Delonix regia, 31-XII-1982, O. Yano 5107 p.p. (SP172553).

Distribuição no Brasil: AC, AM, BA, CE, DF, ES, FN, GO, MG, MS, MT, PB, PE, PR, RJ, RS, SC, SE e SP. Associada a Calymperes palisotii Schwaegr.

Frullania gibbosa Nees in Montagne, Ann. Sci. Nat. Bot. ser. 2, 14: 333. 1840.

Material examinado: estrada do norte, ca. $10 \mathrm{~km}$ da cidade, sobre tronco vivo de Leguminosae, 31-XII-1982, O. Yano 5112 (SP181039).

Distribuição no Brasil: AC, AM, AP, BA, DF, ES, GO, MG, MS, MT, PA, PB, PE, PR, RJ, RR, SC e SP.

Frullania glomerata (Lehm. \& Lindenb.) Nees \& Mont., Ann. Sci. Nat. Bot. ser. 2, 9: 46. $1838 \equiv$ Jungermannia glomerata Lehm. \& Lindenb., Ann. Sci. Nat. Bot., sér. 2, 9: 46. 1838.

Material examinado: sobre pedras grandes a $50 \mathrm{~m}$ da água do mar, 29-IX-1977, O. Yano 856 (SP132610).

Distribuição no Brasil: BA, CE, DF, GO, MG, MS, MT, PR, RJ, RS e SP.

Frullania neesii Lindenb. in Gottsche et al., Syn. Hepat.: 450. 1845.

Material examinado: sobre tronco, 29-1X-1977, $O$. Yano 855 p.p. (SP136101).

Distribuição no Brasil: AC, AM, BA, CE, DF, ES, GO, MG, MT, PB, PE, PR, RJ, RR, RS, SE e SP. Associada a Calymperes palisotti Schwägr. e Schiffneriolejeunea polycarpa (Nees) Gradst.

\section{LEJEUNEACEAE}

Bryopteris diffusa (Sw.) Nees in Gottsche et al., Syn. Hepat.: 286. 1845 三 Jungermannia diffusa Sw., Nova Gen. Spec. Plant. Prodr.: 144. 1788.
Material examinado: estrada do sul, ca. $20 \mathrm{~km}$ da cidade, sobre pedras grandes, junto ao rio, 31-XII-1982, O. Yano 5162 (SP181068).

Distribuição no Brasil: AC, AL, AM, BA, CE, ES, MG, MT, PA, PE, PR, RJ, RS, SC, SE e SP.

Bryopteris fruticulosa T. Taylor, London J. Bot. 5: 382.1846.

Material examinado: cachoeira da Toca, sobre pedra úmida, 31-XII-1982, O. Yano 5132 (SP181053); idem, 31-XII-1982, O. Yano 5145 p.p. (SP181059); idem, O. Yano 5160 (SPI81064).

Distribuição no Brasil: AL, AM, BA, DF, ES, GO, MG, MS, MT, PA, PE, PR, RJ, RS, SC, SE e SP. Associada a Plagiochila patentissima Lindenb.

Ceratolejeunea cornuta (Lindenb.) Steph., Pflanzenwelt Ost-Afrikas. 65. 1895 三 Lejeunea cornuta (Lindenb.) Lindenb. in Gotsche Lindenb. \& Nees, Syn. Hepat.: 399. 1845.

Material examinado: estrada do sul, ca. $10 \mathrm{~km}$ da cidade, sobre pedra exposta ao sol, 31-XII-1982, $O$. Yano 5154 (SP181063).

Distribuição no Brasil: AC, AM, AP, BA, CE, MG, PA, PE, PR, RJ, RO, RR, SC e SP.

Cheilolejeunea rigidula (Mont.) R.M. Schust., Castanea 36: 102. 1971 = Lejeunea rigidula Mont., Ann. Sci. Nat. Bot. ser. 2, 14: 336. 1840.

Material examinado: cachoeira da Toca, sobre pedra úmida, 31-XII-1982, O. Yano 5126 p.p. (SP181049); idem, 31-XII-1982, O. Yano 5153 (SP181062).

Distribuição no Brasil: AC, AL, AM, AP, BA, CE, DF, ES, GO, MG, MS, MT, PA, PB, PE, PR, RJ, RR, SC, SE e SP. Associada a Groutiella apiculata (Hook.) H. Crum. \& Steere.

Lejeunea cancellata Nees \& Mont. in Ramón de la Sagra, Hist. Phys. Bot. Plant. Cell. Cuba 9: 472. 1842.

Material examinado: caminho para cachoeira da Toca, sobre pedra, na mata úmida, 31-XII-1982, O. Yano 5122 (SP181046).

Distribuição no Brasil: AL, BA, CE, ES, MS, MT, RJ, SC e SP.

Lejeunea flava (Sw.) Nees, Naturgesch. Eur. Leberm. 3: 277.1838 = Jungermannia flava Sw., Nova Gen. Spec. Plant. Prodr.: 144. 1788. 
Material examinado: estrada perto do riacho Grande, no barranco úmido junto da estrada, 27-VII-983, $O$. Yano et al. 7963 p.p. (SP189412); idem, sobre tronco de árvore viva, mata ciliar, 27-VII-1983, O. Yano et al. 7964 (SP189413).

Distribuição no Brasil: AC, AM, BA, CE, DF, ES, GO, MG, MS, MT, PA, PB, PE, PR, RJ, RR, RS, SC, SE e SP. Associada a Sematophyllum subpinnatum (Brid.) Britt.

Lejeunea laetevirens Nees \& Mont. in Ramón de la Sagra, Hist. Phys. Bot. Plant. Cell. Cuba 9: 469. 1842.

Material examinado: praça Coronel Julião Moura Negrão, sobre tronco de Tipuana tipu, 31-XII-1982, O. Yano 5108 (SP181038).

Distribuição no Brasil: AC, AL, AM, AP, BA, CE, DF, ES, FN, GO, MG, MS, MT, PA, PB, PE, RJ, RR, SC, SE e SP.

Lejeunea phyllobola Nees \& Mont. in Ramón de la Sagra, Hist. Fis. Pol. Natur. Bot. Cuba 9: 471. 1842.

Material examinado: estrada sul, sobre tronco de jaqueira perto da estrada, Praia Grande, 29-IX-1977, O. Yano 859 p.p. (SP132611); idem, cachoeira da Toca, epifilas, 31-XII-1982, O. Yano 5142 (SP181058).

Distribuição no Brasil: AC, AL, AM, BA, CE, DF, ES, GO, MS, MT, PA, RJ, RS, SC e SP. Associada a Schiffneriolejeunea polycarpa (Nees) Gradst.

Leptolejeunea elliptica (Lehm. \& Lindenb.) Schiffn. in Engler \& Prantl, Natürl. Pflanzenfam. 1(3): 126. 1893.

Material examinado: cachoeira da Toca, epifilas, 31-XII-1982, O. Yano 5127 (SP181050).

Distribuição no Brasil: AC, AL, AM, AP, BA, CE, DF, ES, GO, MG, MT, PA, PE, PR, RJ, RR, SC, SE e SP.

Lopholejeunea nigricans (Lindenb.) Schiffn., Conspec. Hepat. Archip. Ind.: 293.1898 इLejeunea nigricans Lindenb., Syn. Hepat. 316. 1845.

Material examinado: caminho pra cachoeira da Toca, sobre pedra, margem do riacho, 31-XII-1982, O. Yano 5116 (SP181040); idem, estrada sul, ca. $20 \mathrm{~km}$ da cidade, sobre pedras junto da cachoeira, 31-XII-1982, O. Yano 5161 (SP181065); idem, sobre pedra submersa na água do rio, 31-XII-1982, O. Yano 5165 (SP181067).
Distribuição no Brasil: AC, AM, BA, CE, DF, ES, GO, MG, MS, MT, PA, PE, PR, RJ, SC e SP.

Lopholejeunea subfusca (Nees) Schiffn., Bot. Jahrb. Syst. 23: 593. 1897 EJungermannia subfusca Nees, Enum. Plant. Crypt. Jav.: 36. 1830.

Material examinado: caminho para cachoeira da Toca, sobre pedra, na mata úmida, 31-XII-1982, O. Yano 5123 (SP181047); idem, sobre pedra úmida junto da cachoeira, 31-XII-1982, O. Yano 5135 (SP181056).

Distribuição no Brasil: AC, AM, AP, BA, CE, DF, ES, GO, MG, MS, MT, PA, PB, PE, RJ, RO, RR, $\mathrm{SC}$ e SP.

Schiffneriolejeunea polycarpa (Nees) Gradst., J. Hattori Bot. Lab. 38: 355. 1974 = Jungermannia polycarpa Nees in Martius, Flora Brasil. enum. plant. 1(1): 350. 1833.

Material examinado: sobre tronco de jaqueira, 29-IX-1977, O. Yano 855 p.p. (SP136101); idem, estrada sul, sobre tronco de jaqueira perto da estrada, praia Grande, 29-IX-1977, O. Yano 859 p.p. (SP132611).

Distribuição no Brasil: AC, AM, BA, CE, DF, ES, GO, MG, MS, MT, PA, PB, PE, RJ, RR, RS, SC, SE e SP. Associada a Calymperes palisotii Schwägr., Frullania neesii Lindenb. e Lejeunea phyllobola Nees \& Mont.

Taxilejeunea pterigona (Lehm. \& Lindenb.) Schiffn. in Engler \& Prantl, Natürl. Pflanzenfam. 1(3): 125. $1893 \equiv$ Jungermannia pterigona Lehm. \& Lindenb. in Lehmann, Nov. Stirp. Pugillus 6: 44. 1834.

Material examinado: cachoeira da Toca, sobre pedra úmida, 31-XII-1982, O. Yano 5129 (SP181052); idem, 31-XII-1982, O. Yano 5152 (SP181061).

Distribuição no Brasil: AM, BA, ES, GO, MG, RJ, SC e SP.

\section{MARCHANTIACEAE}

Dumortiera hirsuta (Sw.) Nees in Martius, Flora Brasil. enum. plant. 1(1): 307. 1833 = Marchantia hirsuta Sw., Nova Gen. Spec. Plant. Prodr.: 145. 1789.

Material examinado: ca. $20 \mathrm{~km}$ da cidade, sobre pedras semicobertas pela água do rio, 31-XII-1982, O. Yano et al. 5168 p.p. (SP181069). 
Distribuição no Brasil: AC, AM, DF, ES, MG, MS, MT, P A, PR, RJ, RS, SC e SP. Associada a Vesicularia vesicularis var. rutilans (Brid.) W.R. Buck.

Marchantia chenopoda L., Sp. Pl. 2: 1137. 1753.

Material examinado: sobre pedras no bordo de um riacho, 17-X-1970, D.M. Vital 1735 p.p. (SP87561).

Distribuição no Brasil: AC, AM, DF, ES, MG, MT, PR, RJ, RS, SC e SP. Associada a Vesicularia vesicularis var. rutilans (Brid.) W. R. Buck.

\section{PALLAVICINIACEAE}

Symphyogyna aspera Steph. ex McCormick, Bot. Gaz. 58: 403. 1914.

Material examinado: caminho para cachoeira da Toca, sobre pedra, margem do riacho, 31-XII-1982, O. Yano 5117 p.p. (SP181041).

Distribuição no Brasil: AM, BA, CE, DF, ES, GO, MG, MS, MT, P A, PE, RJ, RS, SC e SP. Associada a Lophocolea martiana Nees.

\section{PLAGIOCHILACEAE}

Plagiochila disticha (Lehm. \& Lindenb.) Lindenb., Spec. Hepat. 4: 108. $1840 \equiv$ Jungermannia disticha Lehm. \& Lindenb. in Lehmann, Nov. Stirp. Pugillus 6: 64. 1834.

Material examinado: cachoeira da Toca, sobre pedra úmida, 31-XII-1982, O. Yano 5133 (SP181054).

Distribuição no Brasil: AC, AL, AM, AP, BA, CE, DF, ES, GO, MG, MS, MT, P A, PB, RJ, RR, RS, $\mathrm{SC}$ e SP.

Plagiochila martiana (Nees) Lindenb., Spec. Hepat. 1: 12.1839 = Jungermannia martiana Nees, Linnaea 6: 617. 1831.

Material examinado: caminho para cachoeira da Toca, sobre pedra, na mata úmida, 31-XII-1982, O. Yano 5121 (SP181045).

Distribuição no Brasil: AC, AL, BA, CE, DF, ES, GO, MG, MS, MT, PA, PE, PR, RJ, RS, SC e SP.

Plagiochila patentissima Lindenb., Spec. Hepat.: 64. 1840 .

Material examinado: cachoeira da Toca, sobre pedra úmida, 31-XII-1982, O. Yano 5128 (SP181051); idem, 31-XII-1982, O. Yano 5145 p.p. (SP181059).
Distribuição no Brasil: ES, MG, PR, RJ, RS, SC e SP. Associada a Bryopteris fruticulosa $\mathrm{T}$. Taylor.

Plagiochila rutilans Lindenb., Spec. Hepat. fasc. 2-4: 47.1841.

Material examinado: caminho para cachoeira da Toca, sobre pedra, na mata úmida, 31-XII-1982, O. Yano 5120 (SP181044); idem, O. Yano 5134 p.p. (SP181055); idem, 31-XII-1982, O. Yano 5140 (SP181057).

Distribuição no Brasil: AC, AM, AP, BA, CE, ES, MG, MT, PA, PE, RJ, RR, RS, SC e SP. Associada a Trichocolea tomentosa (Sw.) Gottsche.

\section{RADULACEAE}

Radula cubensis Yamada, J. Hattori Bot. Lab. 54: 241. 1983.

Material examinado: caminho para Cachoeira da Toca, sobre pedra, na mata úmida, 31-XII-1982, O. Yano 5119 (SP181043).

Distribuição no Brasil: BA, ES, SC e SP.

Radula javanica Gottsche in Gottsche et al., Syn. Hepat.: 257. 1845.

Material examinado: cachoeira da Toca, sobre pedra úmida, 31-XII-1982, O. Yano 5146 (SP181060).

Distribuição no Brasil: AC, AM, AP, BA, ES, MG, MS, MT, PA, PE, PR, RJ, RS, SC e SP.

Radula nudicaulis Steph., Spec. Hepat. 4: 174. 1910.

Material examinado: cachoeira da Toca, sobre galho de árvore, 31-XII-1982, O. Yano 5125 (SP181048).

Distribuição no Brasil: ES, MG, PR, RJ e SP.

\section{TRICHOCOLEACEAE}

Trichocolea tomentosa (Sw.) Gottsche, Mexik. Leverm.: 119. 1863 इ Jungermmania tomentosa Sw., Nov. Gen. Spec. Plant. Prodr.: 145. 1788.

Material examinado: cachoeira da Toca, sobre pedra úmida, 31-XII-1982, O. Yano 5134 p.p. (SP181055).

Distribuição no Brasil: ES, MG, PR, RS, SC e SP. Associada a Plagiochila rutilans Lindenb. 


\section{Literatura citada}

Ângelo, S. (coord.) 1989. Ilhas do Litoral Paulista. Série Documentos. Secretaria do Meio Ambiente, São Paulo.

Bischler, H. 1969. Le genre Leptolejeunea (Spruce) Steph. en Amérique. Nova Hedwigia 17: 265-350.

Buck, W.R. 1998. Pleurocarpous mosses of the West Indies. Memoirs of The New York Botanical Garden 82: 1-400.

Buck, W.R. \& Goffinet, B. 2000. Morphology and classification of mosses. In: A.J. Shaw \& B. Goffinet (eds.). Bryophyte Biology. Cambridge University Press, Cambridge, pp. 71-123.

Churchill, S.P. \& Linares C., E.L. 1995. Prodromus Bryologiae Novo-Granatensis: Introduccion a la flora de musgos de Colombia. Biblioteca Jose Jeronimo Triana 12: 1-924.

Crandal-Stotler, B. \& Stotler, R. 2000. Morphology and classification of Marchantiophyta. In: A.J. Shaw \& B. Goffinet (eds.). Bryophyte Biology. Cambridge University Press, Cambridge, pp. 21-70.

Florschuitz, P.A. 1964. The mosses of Suriname. Part 1. E.J. Brill, Leiden.

Fulford, M.H. 1968. Manual of the Leafy Hepaticae of Latin American 3. Memoirs of The New York Botanical Garden 11: 277-392.

Fulford, M.H. 1976. Manual of the Leafy Hepaticae of Latin America 4. Memoirs of The New York Botanical Garden 11: 393-535.

Gradstein, S.R. 1994. Lejeuneaceae. Ptychantheae, Brachiolejeuneae. Flora Neotropica Monograph 62: 1-216.

Gradstein, S.R. \& Costa, D.P. 2003. The Hepaticae and Anthocerotae of Brazil. Memoirs of The New York Botanical Garden 87: 1-318.

Joyce, M.V., Mello, Z.R. \& Yano, O. 2006. Briófitas da Ilha das Palmas, Guarujá, São Paulo, Brasil. Boletim do Instituto de Botânica 18: 101-109.
La Farge, C., Mishler, B.D., Wheeler, J.A., Wall, D.P., Johannes, K., Schaffer, S. \& Shaw, A.J. 2000. Phylogenetic relationships within the Haplolepideous Mosses. The Bryologist 103: 257-276.

Oliveira-e-Silva, M.I.M.N. \& Yano, O. 2000. Musgos de Mangaratiba e Angra dos Reis, Rio de Janeiro, Brasil. Boletim do Instituto de Botânica 14: 1-138.

Peralta, D.F. 2005. Musgos (Bryophyta) do Parque Estadual da Ilha Anchieta (PElA), São Paulo, Brasil. Dissertação de Mestrado, Instituto de Botânica, São Paulo.

Reese, W.D. 1993. Calymperaceae. Flora Neotropica Monograph 58: 1-102.

Schuster, R.M. 1980. The Hepaticae and Anthocerotae of North America east of the hundredth meridian. Columbia University Press, New York, v. 4, pp. 1-1334.

Sharp, A.J., Crum, H. \& Eckel, P.M. 1994. The moss flora of Mexico. Memoirs of The NewYork Botanical Garden 69: 1-1113.

Stotler, R. \& Crandal-Stotler, B. 2005. A Revised Classification of the Anthocerotophyta and a Checklist of the Hornworts of North America, North of Mexico. The Bryologist 108: 16-26.

Visnadi, S.R. \& Vital, D.M. 2001. Briófitas das Ilhas de Alcatrazes, do Bom Abrigo, da Casca e do Castilho, Estado de São Paulo, Brasil. Acta Botanica Brasilica 15: 255-270.

Yano, O. 1990. Musgos do Parque Estadual da Ilha do Cardoso, estado de São Paulo: Sphagnaceae, Rhizogoniaceae, Mniaceae, Racopilaceae e Phyllogoniaceae. ACIESP 71: 411-438.

Yano, O., Mello, Z.R. \& Colletes, A.G. 2003. Briófitas da Ilha Urubuqueçaba, Santos, São Paulo, Brasil. Iheringia, série Botânica 58: 195-214.

Yano, O. \& Peralta, D.F. 2007. Musgos (Bryophyta). In: J.A. Rizzo (coord.). Flora dos Estados de Goiás e Tocantins: Criptógamos. Publicação da Unidade de Conservação, Pró Reitoria de Pós Graduação, Universidade Federal de Goiás, Goiás, v. 6, pp. 1-333. 